Revue

d'ethnoécologie
Revue d'ethnoécologie

12 | 2017

La Poule. Pratiques d'élevage et histoire culturelle

\title{
Les races de poules
}

Formation, évolution, présentation générale

Poultry breeds: Creating process, evolution, overview

\section{Bernard Denis}

\section{(2) OpenEdition}

\section{Journals}

Édition électronique

URL : http://journals.openedition.org/ethnoecologie/3331

DOI : 10.4000/ethnoecologie.3331

ISSN : 2267-2419

Éditeur

Laboratoire Eco-anthropologie et Ethnobiologie

Référence électronique

Bernard Denis, «Les races de poules », Revue d'ethnoécologie [En ligne], 12 | 2017, mis en ligne le 18 décembre 2017, consulté le 10 décembre 2020. URL : http://journals.openedition.org/ethnoecologie/ 3331 ; DOI : https://doi.org/10.4000/ethnoecologie.3331

Ce document a été généré automatiquement le 10 décembre 2020.

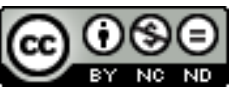

Revue d'ethnoécologie est mis à disposition selon les termes de la licence Creative Commons Attribution - Pas d'Utilisation Commerciale - Pas de Modification 4.0 International. 


\title{
Les races de poules
}

\author{
Formation, évolution, présentation générale \\ Poultry breeds: Creating process, evolution, overview
}

\section{Bernard Denis}

1 Si l'expression classique est celle de "races de poules", il convient de signaler que, zoologiquement, le nom officiel de l'espèce est "Coq domestique ». Formellement, on devrait donc parler de "races de coqs». Le Coq domestique dérive de l'espèce Gallus gallus, le Coq bankiva (ou Coq doré, ou Coq rouge) du Sud-Est asiatique. On admettait classiquement que la domestication avait eu lieu 3000 avant J.-C. Mais de nouvelles données indiquent qu'elle est plus ancienne, ayant débuté vers -4500 ans dans la vallée de l'Indus et peut-être même vers -8000 en Asie sud-orientale. L'espèce domestique s'est répandue dans le monde entier grâce aux mouvements de populations humaines et au commerce antique.

2 Nous évoquerons pour commencer le classement traditionnel des races de poules en deux grands groupes, puis nous nous intéresserons à ce que nous enseigne la littérature zootechnique ancienne sur l'histoire des races gallines. L'étude des "variations", morphologiques et physiologiques, qui servent au classement et à la description des races, suivra et précédera la présentation de quelques races.

\section{Les deux « grands groupes » de races gallines}

3 La tradition continue de classer les races de poules en deux groupes principaux :

- les races "européennes", dont on peut retenir les caractéristiques suivantes, étant entendu qu'elles ne se retrouvent pas chez tous les animaux : format moyen, crête simple, tarses généralement gris, oreillons blancs, crâne étroit et long, yeux affleurant la face, ponte d'œufs blancs. La Gauloise est la représentante type ;

- les races "asiatiques", qui se distinguent bien des précédentes: forte taille, crête souvent fraisée, tarses généralement jaunes, oreillons rouges, crâne court et large, yeux enfoncés sous une arcade sourcilière proéminente, œufs teintés. L'exemple type est la Brahma-Pootra. 
De nombreuses races "synthétiques » ont été créées par croisement entre les deux groupes, les races asiatiques les plus utilisées en Occident ayant été la Cochin et la Brahma au départ, puis la Langshan. Il en est résulté que ces races d'origine métisse sont devenues plus nombreuses que celles d'Orient ou d'Occident. Le but des croisements fut surtout d'augmenter le potentiel de production zootechnique (ponte, croissance), d'autres objectifs ayant toutefois été également visés: le combat et l'agrément (ornement, prestige...).

5 Il existe un très grand nombre de races de poules dans le monde mais quelques-unes seulement se sont imposées dans le secteur industriel, où elles sont utilisées en croisement. L'immense majorité demeure locale; elle est entretenue par de nombreux amateurs.

\section{Les populations « traditionnelles » de poules}

6 Les diverses «Maison Rustique " qui ont été publiées à partir du XVI ${ }^{\mathrm{e}}$ siècle, y compris celle du XIX ${ }^{e}$ siècle, font à peine allusion à des populations pouvant avoir valeur de races. Pourtant, il existait obligatoirement des populations régionales dont seront issues les futures « races standardisées», qu'elles gardent le type européen ou témoignent d'une forte influence asiatique.

7 Liger (édition consultée : 1768) précise ainsi que « Les poules sont fort différentes suivant les endroits d'où elles viennent et les aliments dont on les nourrit». Il estime que la variation morphologique permet au moins de repérer des individus, et il précise :

- il y a des poules naines en Bretagne, dont la chair est délicate,

- il existe des poules frisées, dont les poussins meurent facilement de froid,

- les poules de Padoue sont plus grosses que les autres,

- les poules de Turquie ont un plumage très beau et très varié,

- en Perse, il y a des poules qui n'ont ni queue ni croupion,

- en Chine, certaines poules ont de la laine semblable à celle des moutons.

On remarque au passage que, au-delà du vocabulaire, certaines mutations étaient parfaitement identifiées (nanisme, plumes frisées, plumes soyeuses, absence de croupion).

9 Sur le plan de leurs qualités, Liger vante les poules de moyenne grandeur et noires car elles ont la chair plus délicate et pondent plus que les autres. Il ajoute que celles qui ont la tête grande, la crête pendante et rouge, les jambes et les pieds jaunes passent aussi pour bonnes et fécondes. Ces propos suscitent deux remarques : les qualités prêtées à tort ou à raison aux poules noires pourraient expliquer qu'elles se soient développées par la suite au point que cette couleur est volontiers associée aujourd'hui à nos vieilles races autochtones; le fait que le noir, au XVIII siècle, n'était semble-t-il qu'une couleur parmi d'autres et, par ailleurs, l'existence de tarses jaunes bien avant que des croisements ne $\mathrm{s}^{\prime}$ opèrent avec des races asiatiques ${ }^{1}$ prouvent que les populations loco-régionales étaient polymorphes au niveau de la coloration. Liger signale par ailleurs que certaines régions sont réputées pour la taille et la délicatesse de la chair de leurs volailles : le pays de Caux, la région du Mans, celle de Barbezieux (Lodunois).

Dans la Maison rustique du XIX ${ }^{e}$ siècle (1837), on trouve mention seulement de la «poule ordinaire », habituellement rouge-brun mais qui est également de «toutes les nuances", la « poule anglaise » (naine), les " poules russes », encore appelées « poules américaines » 
ou «poules de Padoue» (!), aux pattes longues et vigoureuses, à chair fine et pondant bien.

11 Manifestement, il faudra aller plus loin dans le $\mathrm{XIX}^{\mathrm{e}}$ siècle pour que l'on commence à parler de races avec rigueur. Nous retiendrons un ouvrage fondamental, celui de Charles Jacque.

\section{L'ouvrage Le Poulailler, de Charles Jacque (1858)}

Charles Jacque est un peintre de l'École de Barbizon, qui s'est particulièrement fait connaître pour ses peintures de moutons. Mais il était par ailleurs passionné de poules et souhaitait illustrer un livre qui leur aurait été consacré. L'occasion ne s'étant pas présentée, il décida de l'écrire lui-même, en le voulant avant tout pratique, faisant bénéficier les lecteurs de sa propre expérience en élevage. Par ailleurs, il l'a naturellement illustré.

On voit apparaître dans son livre un nombre substantiel de races (que l'auteur appelle " espèces", ce qui était encore fréquent), qui sont regroupées en quatre ensembles: grandes espèces indigènes, grandes espèces exotiques, espèces moyennes dites d'agrément, espèces naines.

Les «grandes espèces indigènes " comprennent trois races françaises (Houdan, Crèvecœur, La Flèche), une anglaise (Dorking), une espagnole (Espagnole), une hollandaise (Bréda), une belge (race de Bruges). Charles Jacque ajoute que les espèces françaises de Caux, de Caumont, du Mans, de Barbezieux, de Bresse, de Rennes, d'Angers, d'Argentan, etc. ne sont que des variétés des trois espèces principales françaises. On voit, au total, apparaitre un grand nombre de populations qui étaient ignorées auparavant.

Nous passerons sur les races moyennes dites de luxe ou d'agrément et sur les races naines, mais nous signalerons les trois races que l'auteur mentionne dans l'ensemble "grandes espèces exotiques": la race de Cochinchine (ou de Chang-Haï, d'où elle provenait en réalité), la Brahma-Pootra et la Malaise. Charles Jacque est l'un des premiers en France à avoir pu se procurer des sujets de ces races et les décrire. Elles sont à l'origine, en France, d'un ample processus de croisements avec les races indigènes, sur le modèle de ce qui se passait déjà en Angleterre et qui a été appelé la « Cochinmania ».

\section{Influence des races asiatiques : la « Cochinmania »}

C'est dans la première moitié du XIX ${ }^{\mathrm{e}}$ siècle, spécialement à partir de 1840, que survint en Europe un évènement majeur pour les races avicoles : l'arrivée de "géantes " asiatiques (Cochinchinoise, puis Brahma et, plus tard, Langshan) et le succès que leur conféra l'engouement des grands de ce monde. En 1846, la reine Victoria en exposa à Dublin et, vers 1860, l'impératrice Eugénie fit de même en France. La bourgeoisie ne tarda pas à l'imiter et, peu à peu, tous les éleveurs finirent par suivre le mouvement. Il en résulta que les races indigènes furent largement croisées avec des races asiatiques, ce qui donna naissance à un certain nombre de races prestigieuses (Sussex, Orpington...) et, le mouvement ayant concerné également l'Amérique du Nord, la plupart des races « américaines » : Wyandotte, New Hampshire, Plymouth Rock, Rhode Island, etc. 

signaler qu'elle n'était sans doute pas la première. Au cours des siècles précédents, il est en effet probable que des volailles asiatiques n'ayant pas été consommées pendant les voyages maritimes se soient retrouvées dans les basse-cours européennes. On en parle pour la Marans, certains voyant là l'origine de la coloration particulière, brun-chocolat, de ses œufs; on en parle également pour les poules de Barbezieux, de La Flèche et du Mans.

Passé le cap de la curiosité, l'intérêt zootechnique des races asiatiques est ressorti rapidement. Plus lourdes (on les qualifiait de « géantes»), mieux conformées, meilleures pondeuses en raison d'une ponte plus étalée dans l'année, elles disposaient des qualités requises pour améliorer les productions de viande et d'œufs.

Au total, les races actuelles résultent :

- d'une sélection de races locales (ayant pu toutefois connaître quelque retrempe avec du sang asiatique). C'est leur poids, moyen, qui traduit le mieux leur origine ancienne ;

- de croisements de races locales avec des races asiatiques (ce sont de loin les plus nombreuses);

- de croisements divers, simples ou complexes.

Des populations naines sont connues depuis longtemps. Par croisement, elles ont servi à nanifier beaucoup de races. Au début du xx ${ }^{e}$ siècle, les Anglais les appelaient volontiers Bantam (mot qui signifie « coq nain » mais est aussi le nom d'une ville de l'île de Java). Le nom est resté et le Bantam Club correspond donc au Club des races naines qui, en France, rassemble les éleveurs de vingt-trois races naines.

21 Envisageons à présent les bases de la classification des races qui, mis à part l'ordre alphabétique, simple mais n'ayant aucune signification scientifique, comprennent essentiellement les variations, morphologiques surtout, susceptibles d'être observées. Un classement phylogénétique apparait bien difficile compte tenu des croisements auxquels nous venons de faire allusion.

\section{Les variations chez la Poule}

Selon une tradition remontant au début $\mathrm{du} \mathrm{xx}^{\mathrm{e}}$ siècle, il est commode de retenir le système de classement dit des "coordonnées baroniennes ", qui distingue la "plastique " (en gros, la forme), la "phanéroptique " (ou "décor de la forme») et l'« énergétique » (essentiellement les aptitudes) ${ }^{3}$.

Pour caractériser la forme d'un animal, on s'intéresse à ses proportions (il est longiligne, ou bréviligne, ou médioligne), son profil (convexiligne, concaviligne, rectiligne), son format (hypermétrique, hypométrique, eumétrique). Si le profil s'apprécie très mal chez les volailles, on repère aisément des sujets longilignes (animaux allongés, étroits, fins, aux tarses relativement longs), des sujets brévilignes (animaux ramassés, larges, aux tarses relativement courts), beaucoup de poules se rangeant plutôt dans la catégorie des médiolignes. Les types extrêmes sont spectaculaires, avec par exemple le Combattant anglais d'exposition pour les longilignes et le Combattant indien pour les brévilignes. Moins accusés, les types longilignes classiques se rencontrent dans les races spécialisées pour la ponte et les brévilignes dans les races de chair. Le format, qui résulte formellement de la hauteur, du poids et du volume, peut être approché simplement par le poids chez les volailles. La moyenne se situe, pour les poules 
à 2-2,5 kg, pour les coqs à 3-3,5 kg. Les races lourdes (« hypermétriques ») sont à 2,5-3,5 et 3,5-4. Les races légères (" hypométriques ») se situent en dessous des valeurs moyennes. La caractérisation de la forme doit être complétée par la prise en compte d'éventuelles particularités, qui peuvent modifier la silhouette. Il en est ainsi de la forme de la crête, très diverse, du nombre de doigts (normalement quatre mais il existe des races pentadactyles), de la très rare absence de croupion. On connaît également des cas de véritable "bassétisme ", c'est-à-dire de raccourcissement des membres. Les 'bassets' sont surtout connus chez le chien mais ils existent aussi dans d'autres espèces.

Ce sont les caractéristiques du plumage qui composent la phanéroptique.

Celui-ci varie :

- dans sa texture : les plumes sont lisses (le plus souvent), frisées, soyeuses...

- son étendue : les tarses sont habituellement nus mais ils peuvent être emplumés ; le plus souvent absents, on peut noter la présence de huppe et/ou de favoris; on connaît également une mutation « cou nu »;

- sa couleur : il existe de très nombreux patrons de coloration, dont la présentation est impossible dans les limites de cet article. Signalons seulement qu'ils se ramènent à cinq couleurs de base : blanc, noir, rouge, jaune (ou fauve), bleu gris (ou bleu ardoisé), mais avec beaucoup de variations de nuances qui portent un nom propre à chacune. Ces couleurs peuvent se rencontrer seules sur l'ensemble du plumage ou se combiner de diverses manières, ce qui donne des plumages : maillé, pailleté, barré, crayonné, herminé, etc...

L'énergétique concerne les qualités zootechniques de production. Contentons-nous de signaler que les meilleures races de ponte (et, surtout, leurs «hybrides" issus de croisements industriels) produisent environ 300 œufs par an. Les races locales se situent dans une fourchette de 50 à 150, mais certaines races fermières dépassent nettement ces valeurs. La vitesse de croissance est très variable : en moyenne, ce sont les races les plus lourdes qui croissent le plus rapidement mais, dans le secteur industriel, la sélection sur ce critère a modifié les performances de manière spectaculaire.

\section{Évocation de quelques races}

Nous parlons volontairement d'évocation et non pas de présentation, qui demanderait une étude spéciale. Notre propos est seulement de mettre en place quelques noms de races et de situer leur importance. Les ouvrages de référence ne manquent pas en langue française, écrits notamment par Jean-Claude Périquet ${ }^{4}$. On trouvera par ailleurs sur Internet de nombreuses photographies de qualité de toutes les races de poule.

Pour son importance historique et comme exemple des races asiatiques qui ont marqué les races occidentales, on peut citer la Brahma-Pootra, qui conserve aujourd'hui une certaine importance chez les amateurs. Il convient ensuite de mentionner les quelques races qui se sont imposées dans le secteur industriel. Elles sont au nombre de quatre. La Leghorn, issue de la sélection américaine d'une race italienne, est à l'origine de tous les œufs blancs qui se consomment dans le monde. La Rhode Island est une productrice d'œufs colorés, certains pays, dont la France, ayant résisté aux œufs blancs que voulaient pourtant imposer les sélectionneurs internationaux. Dans le secteur de la chair, la White Rock (ou Plymouth Rock blanche) s'est avérée être la meilleure pour être croisée avec la Cornish. Celle-ci résulte d'une sélection effectuée par Charles Vantress à partir du 
Combattant indien, que nous avons déjà évoqué. Ces quatre races - deux pour la ponte, deux pour la chair - ne sont évidemment pas élevées en pure race; elles ont servi à constituer des souches, pures ou elles-mêmes « synthétiques ", qui sont utilisées dans des schémas de croisements complexes, dits industriels. Ce sont les mêmes populations et les mêmes croisements qui, dans le secteur industriel, se sont imposés dans le monde entier. Bien entendu, les sélectionneurs peuvent avoir, ponctuellement, utilisé d'autres races dans leurs schémas de croisements, mais l'information n'est connue que d'eux seuls.

On pourrait ensuite citer quelques autres grandes races internationales, qui étaient très réputées comme races fermières, dotées de bonnes aptitudes mixtes, et que l'on avait d'ailleurs tenté de placer dans les schémas de croisements des débuts de l'aviculture industrielle, mais sans succès. Dans le même temps, elles demeuraient très appréciées dans les basse-cours fermières. Celles-ci ayant beaucoup perdu de leur importance, les races concernées se sont elles aussi réfugiées chez les amateurs. Nous n'en citerons qu'une seule, très connue et appréciée il y a une cinquantaine d'années, dont le plumage herminé avait laissé croire que c'était la couleur type des poules. Il s'agit de la Sussex.

Citons enfin quelques races françaises. La seule qui ait conservé une réelle importance économique est la poule de Bresse, qui n'a le droit de porter ce nom que dans l'aire de production du poulet de Bresse AOC. Ailleurs, elle s'appelle Gauloise. Elle est de format moyen et a donc été relativement peu marquée par le sang asiatique. D'anciennes races locales tentent de créer un marché, le plus souvent régional, de poulets hauts de gamme, sur le modèle du poulet de Bresse. La Coucou de Rennes y est semble-t-il parvenue et d'autres suivent le même chemin, notamment la Noire du Berry, à laquelle la Fondation du Patrimoine a attribué son prix pour l'agrobiodiversité animale il y a quelques années.

L'étude des races de poules ne manque pas d'intérêt, sur le plan historique notamment, pour les amateurs mais aussi les zootechniciens. En effet, si l'évolution des races animales répond, en élevage, à un certain schéma-type valable pour toutes les espèces, deux éléments ont été exacerbés chez la poule: les croisements réalisés au xIX siècle; l'expansion des races les plus performantes au $\mathrm{xx}^{\mathrm{e}}$ siècle. Quatre d'entre elles seulement se sont imposées au plan mondial! Notons, en parallèle, le rôle joué par les amateurs dans la conservation de la variabilité génétique et, plus récemment, sur le modèle du poulet de Bresse, quelques efforts de valorisation économique de certaines races anciennes.

\section{BIBLIOGRAPHIE}

Bixio A. (Ed.) 1837 - Maison Rustique du XIXe siècle, Encyclopédie d'Agriculture pratique. II, Cultures industrielles et animaux domestique. Paris, au Bureau, Quai aux Fleurs, $\mathrm{n}^{\circ} 15$.

Blanchon H.-L. \& comte Delamarre de Monchaux 1924 - Toutes les poules et leurs variétés. ParisBruxelles-Madrid, Charles Amat/Éd. Marette/Rivas Grande, 644 p.

Bréchemin L. 1920 - La Basse-cour productive. Monographie des races de poules domestiques et d'agrément. Paris, Librairie agricole de la Maison Rustique, 376 p. 
Jacque C. 1858 - Le Poulailler. Monographie des poules indigènes et exotiques. Paris, Librairie agricole de la Maison Rustique, 360 p.

Liger L. 1768 - La Nouvelle Maison Rustique ou Économie générale de tous les biens de la campagne, augmentée et mise en meilleur ordre par M... Paris, Chez la veuve Savoye.

Périquet J.-C. 1994 - Le Grand livre des volailles de France. Races anciennes, rares, disparues ou actuelles. Paris, Éditions Rustica, 152 p.

Périquet J.-C. 2012 - Le Traité Rustica de la basse-cour. Paris, Éditions Rustica, 560 p.

Voitellier C. 1918 - Aviculture. Paris, J.-B. Baillière et Fils, 516 p. (Encyclopédie agricole)

\section{NOTES}

1. En réalité, comme on le verra plus loin, il n'est pas impossible que des croisements avec des sujets de races asiatiques se soient ponctuellement déjà produits. Cela n'enlève toutefois rien à l'idée que nos populations loco-régionales étaient de couleurs très variées.

2. Raoul Baron fut professeur de zootechnie à l'École vétérinaire d'Alfort à la fin du XIXe et au début $\mathrm{du} \mathrm{xx}^{\mathrm{e}}$ siècle.

3. En dehors de la prise en compte des variations, il est possible de recourir à deux autres systèmes de classement rationnels des races de poules : l'origine géographique (qui n'est pas sans rapport avec la phylogenèse supposée) et les aptitudes, les races étant classées en races de ponte, de chair, ou mixtes.

4. J.-C. Périquet a écrit de nombreux ouvrages, certains ne traitant que des races, d'autres des diverses facettes de l'aviculture familiale. Nous faisons figurer deux des plus importants en bibliographie.

\section{RÉSUMÉS}

L'auteur entend se limiter à une étude très générale. Il signale tout d'abord le classement traditionnel des races de poules en races européennes et asiatiques, puis évoque quelques faits historiques importants. En France, la littérature agronomique est discrète sur les races de poules, jusqu'à la parution de l'ouvrage fondamental de Charles Jacque, Le Poulailler, en 1858. L'introduction de races asiatiques en Europe, qui commença en Angleterre, eut une importance considérable : plus lourdes et plus productives que les races autochtones, elles furent largement croisées avec ces dernières et de nouvelles populations en ressortirent. Les variations, morphologiques surtout, qui sont l'un des moyens de classer les races, sont brièvement présentées. Enfin, quelques races sont évoquées à titre d'exemple, dont les quatre « grandes » qui se sont imposées dans le secteur industriel mais sont utilisées en croisements : Leghorn, Rhode Island, Cornish, White Rock.

The author intends to give out a very general study. First of all, he indicates that hens are traditionally classified into European races and Asian races, and then mentions some important 
historic facts. In France, little can be found on the races of hens in the agronomic literature until the publication of Charles Jacque's fundamental work, Le poulailler, in 1858. The introduction of Asian races in Europe, which began in England, took a noteworthy importance: heavier and more productive than the native races, they were widely crossbred with the latter and new populations stood out from them. The variations, especially morphological, which are one of the means to classify races, are briefly presented. Finally, some races are taken as examples, such as the four "big" which prevail in the branch of industry but are used in crossbreedings: Leghorn, Rhode Island, Cornish, White Rock.

INDEX

Mots-clés : poule, races, histoire, zootechnie

Keywords : hen, races / breed, history, animal breeding

\section{AUTEUR}

\section{BERNARD DENIS}

Professeur honoraire de l'École vétérinaire de Nantes, président de la Société d'Ethnozootechnie

- 5, Avenue Foch, 54200 Toul

denis.brj@wanadoo.fr 